\title{
UK National Vegetation Classification Database
}

\author{
John Rodwell
}

\begin{abstract}
The UK National Vegetation Classification Database (GIVD ID GB-001) holds the encoded data for 35,000 relevés from the UK NVC programme. The NVC is a phytosociological classification of all vegetation types of natural, semi-natural and major artificial habitats in England, Scotland and Wales. It was published as five volumes of British Plant Communities (Rodwell, J.S., editor, Cambridge University Press, 1991-2000) which contain modular community accounts, synoptic tables and distribution maps of available samples.
\end{abstract}

Keywords: artificial habitat; England; phytosociology; Scotland; Wales.

\section{UK National Vegetation Classification Database}

Scope: The UK National Vegetation Classification Database holds the encoded data for 35,000 relevés from the UK NVC programme. The NVC is a phytosociological classification of all vegetation types of natural, semi-natural and major artificial habitats in England, Scotland and Wales. It was published as five volumes of British Plant Communities (Rodwell, J.S., editor, Cambridge University Press, 1991-2000) which contain modular community accounts, synoptic tables and distribution maps of available samples.

Status: finished

Period: $1959-1979$

Database manager(s): John Rodwell (johnrodwell@tiscali.co.uk)

Owner: UK Joint Nature Conservation Committee

Web address: [NA]

Availability: not yet available

Online upload: [NA]

Online search: $[N A]$

Database format(s): MS Access

Export format(s): [NA]

Publication: [NA]

Plot type(s): normal plots

Non-overlapping plots: 35,000

Plot-size range: $1-2,500 \mathrm{~m}^{2}$

Total plot observations: 35,000

Estimate of existing plots: [NA]

Number of sources: 40

Completeness: [NA]

Countries: GB: $100.0 \%$

Forest: [NA] — Non-forest: [NA]

Guilds: all vascular plants: 100\%; bryophytes (terricolous or aquatic): $100 \%$; lichens (terricolous or aquatic): $100 \%$; algae (terricolous or aquatic): $1 \%$

Environmental data: altitude: $40 \%$; slope aspect: $40 \%$; slope inclination: $40 \%$; soil depth: $5 \%$; surface cover other than plants (open soil, litter, bare rock etc.): $5 \%$; soil $\mathrm{pH}: 20 \%$

Performance measure(s): cover: $100 \%$; measurements like diameter or height of trees: $8 \%$

Geographic localisation: small grid (not coarser than $10 \mathrm{~km}$ ): $100 \%$

Sampling periods: $1950-1959: 5.0 \% ; 1960-1969: 10.0 \% ; 1970-1979: 70.0 \%$

Information as of 2012-07-12; further details and future updates available from http://www.givd.info/ID/EU-GB-001

John Rodwell (johnrodwell@ tiscali.co.uk)

Institute of Environmental and Natural Sciences, University of Lancaster, Lancaster LA1 4YQ, UNITED KINGDOM 